\title{
Prevención y tratamiento de la hemorragia gastrointestinal como complicación de la endoscopia terapéutica
}

\author{
Juan Manuel Blancas-Valencia* \\ Gastroenterología, Hospital Ángeles Metropolitano, Ciudad de México, México
}

\section{Introducción}

La endoscopia gastrointestinal en sus inicios era solo diagnóstica, sin embargo, en la actualidad es un procedimiento terapéutico que modifica la historia natural de la enfermedad. Los primeros tratamientos endoscópicos estaban encaminados al control de la hemorragia gastrointestinal. En la actualidad podemos establecer que una de las etiologías que más hemorragia gastrointestinal provoca son los tratamientos endoscópicos de enfermedades no hemorrágicas como por ejemplo la hemorragia pospolipectomía.

Dentro de la endoscopia terapéutica en el tracto gastrointestinal tenemos procedimientos que se pueden considerar como de bajo riesgo y en estos la posibilidad de presentar hemorragia durante el procedimiento 0 después del mismo es en promedio de menor a $1 \%$ y tenemos a la dilatación de estenosis pépticas o posquirúrgicas, la profilaxis primaria y secundaria de la hemorragia Variceal, la gastrostomía endoscópica, la esfinterotomía biliar y la esfinteroplastia. Por otra parte, tenemos procedimientos en donde la hemorragia se puede presentar con mayor frecuencia del 1 a $10 \%$ y es la polipectomía con técnicas avanzadas de resección como son la resección endoscópica de la mucosa y la disección endoscópica de la submucosa en las lesiones mayores de $2 \mathrm{~cm}$, y la dilatación con balón neumático. Otros procedimientos endoscópicos como la colocación de prótesis o el tratamiento de las lesiones hemorrágicas también se pueden complicar con hemorragia, pero su frecuencia es menor al $1 \%$ y en general las recomendaciones que se van a hacer para la prevención y el manejo de la hemorragia de los procedimientos terapéuticos endoscópicos se pueden aplicar a ellos.

También desde el punto de vista del paciente se han identificado factores de riesgo para la presentación de hemorragia post tratamiento endoscópico y se clasifican como riesgo habitual a los pacientes sin comorbilidades y con riesgo aumentado a los pacientes con comorbilidades como insuficiencia hepática, con alteraciones de la coagulación, pacientes con enfermedades cardíacas que toman anticoagulantes orales o que toman aspirina y a mayor número de comorbilidades el riesgo de hemorragia se incrementa, también dentro de los factores a considerar como de riesgo elevado son las lesiones en el colon derecho, las lesiones mayores de $2 \mathrm{~cm}$ en cualquier parte del tracto digestivo y las dilataciones de estenosis localizadas en el intestino delgado.

\section{Prevención}

Para la prevención de la hemorragia se debe realizar en general una evaluación preoperatoria y tener tiempos de coagulación normales y en caso de alteraciones de la coagulación debemos corregirlas, las enfermedades concomitantes deben estar controladas de una manera adecuada, esto es por lo que respecta al paciente y por lo que respecta al médico

\section{Correspondencia:}


y al tratamiento endoscópico de debe seguir la técnica establecida para el procedimiento que se va a realizar.

\section{Tratamiento}

Una vez que se presenta la hemorragia durante el tratamiento endoscópico se debe realizar la hemostasia adecuada para lo cual se debe contar con todos los aditamentos endoscópicos desde la aguja de inyección, los métodos térmicos, clips endoscópicos y de ser posible los polvos hemostáticos, en el caso de lesiones con riesgo de hemorragia elevada se recomienda realizar el procedimiento en un hospital en donde se cuente con el apoyo paraclínico adecuado. Finalmente, al presentarse la hemorragia en forma mediata o tardía se debe someter al paciente a tratamiento endoscópico y se deben tener disponible todos los aditamentos endoscópicos antes mencionados. En general el tratamiento de la hemorragia es exitoso en la mayor parte de los pacientes y se debe realizar en más del $90 \%$ de los casos.

La Dra. Feagins ${ }^{1}$ expone que la hemorragia pospolipectomía ocurre en un 2-3\%. Menciona que el uso profiláctico de los clips en general no es efectivo para los pólipos menores de $1 \mathrm{~cm}$, pero pueden ser efectivos para los pólipos grandes y presenta los resultados de su estudio en donde se establece que los clips no previenen la hemorragia tardía pospolipectomía y logra establecer factores predictivos para hemorragia en los pólipos grandes: 1) dependiente de los pacientes, como son el uso de warfarina con puente de heparina y el uso del clopidogrel y 2) factores endoscópicos, el tamaño del pólipo y la localización proximal. Por otra parte, Shimodate ${ }^{2}$ reportó los resultados de un estudio multicéntrico aleatorizado y abierto realizado en Japón en pacientes sometidos a polipectomía de pólipos de menos de $10 \mathrm{~mm}$ que estaban tomando anticoagulantes orales, los dividió en 2 grupos, uno con puente de heparina y al otro grupo lo mantuvo con su anticoagulación y concluye que retirar los pólipos menores de $10 \mathrm{~mm}$ con asa fría y con terapia de anticoagulación no incrementa los riesgos de hemorragia grave pospolipectomía comparada con los pacientes con heparina y polipectomía con asa caliente, Anderson ${ }^{3}$, recomienda continuar la aspirina en todos los casos de polipectomía, ya sea con técnica habitual o con técnicas avanzadas de resección, también establece que los fármacos antitrombóticos incrementan el riesgo de hemorragia pero no hay suficientes datos para hacer una recomendación de suspender o continuarlos durante el periodo peri polipectomía y si se suspenden deben ser reiniciados lo antes posible después de la colonoscopia.

Raju ${ }^{4}$, menciona que en alrededor de 2 millones de colonoscopias reportadas entre 2001 a 2015 se reportaron en promedio $2.6 \%$ de hemorragia y la mortalidad reportada en un estudio francés por hemorragia fue de 13.2/1,000 procedimientos (Laanani, et al. CGH 2018) y que el uso de clopidogrel no aumento significativamente ni la hemorragia inmediata ni la tardía pos polipectomía, además mostro que el tratamiento profiláctico para prevenir hemorragia con terapia combinada o mecánica no tuvo efectos en la hemorragia tardía en los pólipos pediculados y muestra una tabla para la evaluación del riesgo de hemorragia en donde establece parámetros y da una calificación, muestra 2 clasificaciones, la de Albeniz y la de Bahin, la primera establece como el mayor riesgo de hemorragia cuando hay de 8 a 10 puntos con un $40 \%$ de posibilidades de sangrar, contra un riesgo mínimo si se tienen de 0 a 3 puntos, con un riesgo de 1 a $2 \%$, esto es al momento de realizar la polipectomía. Establece también que para disminuir la hemorragia tardía se debe evaluar muy bien la base de la resección y si se observa un vaso se debe realizar coagulación suave del mismo con el asa de polipectomía o con los fórceps hemostáticos o con los clips. Maranki ${ }^{5}$, muestra una tabla en donde expone los procedimientos endoscópicos con bajo riesgo de hemorragia y procedimientos con alto riesgo de hemorragia, que son los siguientes: polipectomía, el tratamiento de las várices, la enteroscopia profunda terapéutica, la hemostasia endoscópica, la cistogastrostomía, la resección endoscópica de la mucosa, la dilatación neumática de las estenosis, la esfinterotomía biliar, la gastrostomía endoscópica, el ultrasonido endoscópico con toma de biopsia, la ablación de tumores, la disección endoscópica de la submucosa y la yeyunostomía. Pasando a otro procedimiento con riesgo alto de hemorragia Bettenworth $^{6}$, presenta la seguridad y eficacia de la dilatación de las estenosis del intestino delgado en pacientes con enfermedad de Crohn y en 1194 dilataciones realizadas en 468 pacientes se presentaron en total $3,7 \%$ de complicaciones mayores, con un éxito técnico de $88 \%$ y eficacia clínica en el $78 \%$ de los pacientes. No definen el porcentaje de hemorragia ni su manejo, en lo que se refiere a hemorragia por esfinterotomía Giltendez ${ }^{7}$, presenta su experiencia de 5 años en donde se incluyeron 999 pacientes y se 
presento hemorragia en $82(8.2 \%)$ de los cuales 44 fueron inmediatos y 38 tardías, la mayoría de las hemorragias severas fueron de presentación tardía, se realizo hemostasia endoscópica en 71 casos, un paciente se sometió a tratamiento radiológico y no se requirió cirugía. Los factores de riesgo identificados fueron: Cirrosis (OR:7.511) enfermedad renal avanzada (OR:6.634) y precorte (OR:4.190) por lo que se sugiere tener en cuenta estos factores de riesgo para tener mayor y mejor vigilancia en estos casos. La mayoría de los pacientes requiere manejo endoscópico con buenos resultados.

\section{Conflicto de intereses}

No existen conflictos de interés. Sin patrocinio de la industria.

\section{Bibliografía}

1. Feagins $L$, Smith $A, \operatorname{Kim} D$, et al. A prospective, randomized trial of prophylactic hemoclipping for preventing delayed post-polypectomy bleeding in patients with large colonic polyps. DDW congress; 2019 mayo 18-21; San Diego Ca Sesión 2115.

2. Shimodate $\mathrm{Y}$, Takeuchi $\mathrm{Y}$, Mabe $\mathrm{K}$ et al. A multicenter, randomized open Ibel trial comparing heparna bridging with hot snare polypectomy and continuous oral administration of anticoagulants with cold snare polypectomy for patients with colorectal polyps smaller tan $10 \mathrm{~mm}$. Sesión de carteles presentada en DDW; 2019 mayo 18-21; San Diego CA 473.

3. Michelle A Anderson. Polypectomy and anticoagulants/antiplatelets: ¿What should I do? DDW congress; 2019 mayo 18-21; San Diego Ca Sesión 2165.

4. Gottumukkala Raju. Management of complications: Acquired and iatrognic, acute and delayed. DDW congress; 2019 mayo 18-21; San Diego Ca Sesión 2415.

5. Jennifer Maranki. The High risk bleeder: Prevention and Management. DDW congress; 2019 mayo 18-21; San Diego Ca Sesión 5245.

6. Bettenworth D, Bokemeyer A, Kou L, et al, small bowel crohn's disease strictures by balloon-assisted enteroscopy: Pooled análisis of individual data from 210 patients. DDW congress; 2019 mayo 18-21; San Diego Ca poster Mo 1841.

7. Giltendez J, Baguio C, Gabriel E et al. Risk factors, management and outcomes of post endoscopic sphincterotomy bleeding a 5 year single center experience. DDW congress; 2019 mayo 18-21; San Diego Ca Poster sesión Sa 1402. 